\title{
Elucidating the Role of Subdiffusion and Evanescence in the Target Problem: Some Recent Results
}

\author{
E. Abad $^{1} *$ S. B. Yuste ${ }^{2}$, K. Lindenberg ${ }^{3}$ \\ ${ }^{1}$ Departamento de Física Aplicada, Centro Universitario de Mérida, Universidad de Extremadura, \\ E-06800 Mérida, Spain \\ ${ }^{2}$ Departamento de Física, Universidad de Extremadura, E-06071 Badajoz, Spain \\ ${ }^{3}$ Department of Chemistry and Biochemistry, and BioCircuits Institute, University of California San \\ Diego, 9500 Gilman Drive, La Jolla, CA 92093-0340, USA
}

\begin{abstract}
We present an overview of recent results for the classic problem of the survival probability of an immobile target in the presence of a single mobile trap or of a collection of uncorrelated mobile traps. The diffusion exponent of the traps is taken to be either $\gamma=1$, associated with normal diffusive motion, or $0<\gamma<1$, corresponding to subdiffusive motion. We consider traps that can only die upon interaction with the target and, alternatively, traps that may die due to an additional evanescence process even before hitting the target. The evanescence reaction is found to completely modify the survival probability of the target. Such evanescence processes are important in systems where the addition of scavenger molecules may result in the removal of the majority species, or ones where the mobile traps have a finite intrinsic lifetime.
\end{abstract}

Keywords and phrases: diffusion limited reactions, anomalous diffusion, fractional equations

Mathematics Subject Classification: 35K57, 60G22

\section{Introduction}

Since Smoluchowski's seminal work on diffusion-controlled reactions between two species $A$ and $B$ [38], a great deal of work on the subject has populated the literature. Smoluchowski's theory and generalizations thereof have been widely applied in many other fields to successfully compute rates of encounters driven by diffusion. To name but a few, examples include fluorescence and luminescence quenching, reactions between solvated electrons, proton transfer reactions, radical recombination, enzyme-ligand interactions, protein folding, polymer chain growth, and oxygenation-deoxygenation of red blood cells (see eg. [31] and references therein).

In its original version, Smoluchowski's theory deals with two diffusing species $A$ and $B$ which undergo the reaction $A+B \rightarrow$ products upon first encounter (infinite reaction rate). In this framework, the instantaneous reaction between the encountering pair can be modeled by an absorbing boundary condition. The problem of computing the rate of reaction is clearly made difficult because the choice of a specific

*Corresponding author. E-mail: eabad@unex.es 
reaction partner $B$ by an $A$ particle out of a number of $B$ particles which are at comparable distances will affect the chance of reaction and thus the fate of other $A$ particles "competing" for the same $B$. In order to bypass the difficulties arising from statistical correlations between reactant pairs, Smoluchowski assumed that the $B$ species is in vast excess with respect to the $A$ species, implying that the $A$ reactants to a good approximation behave as though they are statistically independent. Under these conditions, the mean concentration of the majority species $B$ can be considered to be constant; the decay of the concentration of the $A$ species is then described by the survival probability $Q_{T}$ of a single $A$ particle ("the target") surrounded by a constant number of $B$ particles (the "traps"), i.e., $c_{A}(t)=c_{A}(0) Q_{T}(t)$. The rate constant $k(t)$ of the reaction can be expressed in terms of $Q_{T}(t)$ as follows [6]:

$$
k(t)=-\frac{d \ln Q_{T}(t)}{d t} \quad \Rightarrow \quad \frac{d Q_{T}(t)}{d t}=-k(t) Q_{T}(t),
$$

thus leading to

$$
Q_{T}(t)=\exp \left[-\int_{0}^{t} k\left(t^{\prime}\right) d t^{\prime}\right] .
$$

Hence the importance of the survival probability problem from the point of view of Smoluchowski's theory.

In the general case, both the target and the traps are assumed to diffuse with diffusion coefficients $D_{A}$ and $D_{B}$, respectively. The computation of the survival probability of the target in this general case is difficult $[8-10,28]$ (in fact, despite many efforts, the full exact asymptotic solution in dimensions $d \geq 3$ is still elusive, see e.g. the conclusions section in [7]). However, it is possible to gain insight into this general problem by considering the limiting cases of an immobile target $\left(D_{A} \rightarrow 0\right)$ or of immobile traps $\left(D_{B} \rightarrow 0\right)$, because they provide bounds on the more general case, bounds that may meet in some situations. These cases are easier to handle analytically. The case $D_{A} \rightarrow 0$ is usually called "the target problem" in the literature, whereas the case $D_{B} \rightarrow 0$ corresponds to the so-called "trapping problem". In the latter case, it can be rigorously shown that the long-time decay of the survival probability of a target surrounded by a sea of uncorrelated traps in a $d$-dimensional Euclidean space follows a stretched exponential law, i.e., $\ln Q_{T}(t) \propto-t^{d /(d+2)}[14]$.

In what follows, we shall focus on the target problem and discuss some recent results which extend available solutions for the case of normal diffusive traps to the case of subdiffusive traps $[7,15,16,18,32,40$ 43]. The motivation for considering subdiffusive motion of the traps stems from the fact that deviations from ordinary diffusion are likely to occur in crowded or complex environments. Such environments frequently arise in biological systems. In our route to the solution, we shall also provide results for the single-trap problem, i.e., the problem of computing the survival probability of the target in the presence of a single trap. We do this for two reasons, namely (i) the solution for the many-trap problem can be constructed from that for the single-trap problem, and (ii) the single-trap system is also interesting in its own right, especially for the study of the geminate recombination problem (for experimental and theoretical studies of this problem see e.g. chapter 6 in Ref. [31] and references therein, as well as Ref. [34] for a recent theoretical study). We shall assume that the motion of the traps is described by a continuous time random walk (CTRW) with a long-tailed waiting time distribution and a jump length distribution of finite variance. In the long time limit this version of the CTRW is equivalent to a fractional diffusion equation [26].

We shall consider two different situations for both the single-trap and the many-trap problems, namely, one where the traps can only die upon interaction with the target, and one where an additional evanescence process is at play $[2,10,19,44]$, that is, the traps may die at a specified rate before reaching the target (in the present work, the evanescence reaction is assumed to take place independently of the CTRW jumps performed by the traps, as opposed to a recently introduced model where disappearance may only take place at the time of each jump [36]). For instance, the presence of scavenger molecules $C$ may result in the removal of $B$ particles before they can reach the target $A[22,31,33]$. Another interesting example is the relaxation of frozen dipoles via collision with diffusing defects $[17,37]$, as the latter may 
coalesce and, consequently, their concentration may decrease in time by mechanisms independent of the interaction with the dipoles. More generally, any process that turns off the interaction between the traps and the target can also be thought of as an evanescence or death process of the traps for the sake of the computation of the survival probability of the target and the associated rate constant of the reaction. As we shall see, the evanescence reaction is found to profoundly modify the survival probability of the target.

The paper is organized as follows. In Sec. 2 we present some recent results for the survival probability of the target in the absence of the evanescence reaction. This presentation includes the case of a partially absorbing target. This is a generalization to a CTRW of Smoluchowski's theory for the case of a finite reaction rate [13]. Next, restricting ourselves to the case of a fully absorbing target, in Sec. 3 we consider the case of evanescent traps. Finally, we conclude with a brief summary in Sec. 4.

\section{The target problem with non-evanescent traps}

\subsection{The single-trap problem}

We start by considering an immobile target of radius $R$ centered at the origin $\mathbf{r}=0$ and a point trap located at an initial position $\mathbf{r}_{0}$. At time $t=0$ the trap starts moving, and if it ever hits the surface of the impenetrable target both the trap and the target disappear. The trap performs a CTRW whose long-time motion is described by the fractional diffusion equation

$$
\frac{\partial w\left(\mathbf{r}, t \mid \mathbf{r}_{0} ; 0\right)}{\partial t}=K_{\gamma} \mathcal{D}_{t}^{1-\gamma} \nabla_{\mathbf{r}}^{2} w\left(\mathbf{r}, t \mid \mathbf{r}_{0} ; 0\right), \quad 0<\gamma \leq 1,
$$

where $w\left(\mathbf{r}, t \mid \mathbf{r}_{0} ; 0\right)$ is the probability density of finding the trap at location $\mathbf{r}$ at time $t$ given that it started at position $\mathbf{r}_{0}$ at $t=0$. The constant $K_{\gamma}$ is the anomalous diffusion coefficient, and $\nabla_{\mathbf{r}}^{2}$ stands for the Laplacian operator with respect to the position $\mathbf{r}$. The initial condition is

$$
w\left(\mathbf{r}, 0 \mid \mathbf{r}_{0} ; 0\right)=\delta\left(\mathbf{r}-\mathbf{r}_{0}\right) .
$$

The Grünwald-Letnikov operator ${ }_{0} \mathcal{D}_{t}^{1-\gamma}$ is defined via the equation

$$
\mathcal{L}_{t \rightarrow u}\left\{{ }_{0} \mathcal{D}_{t}^{1-\gamma} f(t)\right\}=u^{1-\gamma} \mathcal{L}_{t \rightarrow u}\{f(t)\},
$$

where $\mathcal{L}_{t \rightarrow u}\{f(t)\} \equiv f(u)=\int_{0}^{\infty} e^{-u t} f(t) d t$ denotes the Laplace transform (the function and its Laplace transform are clearly distinguished by the argument and so we use the same designation for both). Instead of ${ }_{0} \mathcal{D}_{t}^{1-\gamma}$ we consider the more commonly used Riemann-Liouville fractional derivative ${ }_{0} D_{t}^{1-\gamma}$, defined as follows [30]:

$$
{ }_{0} D_{t}^{1-\gamma} f(r, t)=\frac{1}{\Gamma(\gamma)} \frac{\partial}{\partial t} \int_{0}^{t} d t^{\prime} \frac{f\left(r, t^{\prime}\right)}{\left(t-t^{\prime}\right)^{1-\gamma}} .
$$

When applied to sufficiently smooth functions, the operator ${ }_{0} \mathcal{D}_{t}^{1-\gamma}$ is equivalent to the Riemann-Liouville derivative [30]. The propagator solution of the fractional diffusion equation (2.1) yields a mean square displacement with the long time behavior

$$
\left\langle\left(\mathbf{r}-\mathbf{r}_{0}\right)^{2}\right\rangle \sim K_{\gamma} t^{\gamma}
$$

resulting in subdiffusive behavior when $\gamma$ is less than unity and in normal diffusive behavior when $\gamma=1$.

To complete the calculation of the probability density $w\left(\mathbf{r}, 0 \mid \mathbf{r}_{0} ; 0\right)$ we complement Eq. (2.1) with the boundary conditions

$$
\begin{aligned}
w\left(R, t \mid \mathbf{r}_{0} ; 0\right) & =0, \\
\lim _{r \rightarrow \infty} w\left(\mathbf{r}, t \mid \mathbf{r}_{0} ; 0\right) & =0 .
\end{aligned}
$$


The boundary condition (2.6) implements the fully absorbing property of the target.

We wish to use the solution of this problem to calculate the survival probability $Q_{1, T}\left(\mathbf{r}_{0}, t ; R\right)$ of the target. Note that the survival probability of the target is identical with the survival probability $Q_{1}\left(\mathbf{r}_{0}, t ; R\right)$ of the trap, as we have assumed that both the target and the trap disappear instantaneously upon encounter, i.e., there is only one (shared) decay channel for both particles. Either survival probability is related to the probability density $w\left(\mathbf{r}, t \mid \mathbf{r}_{0} ; 0\right)$ via the equation

$$
Q_{1}\left(\mathbf{r}_{0}, t ; R\right)=\int w\left(\mathbf{r}, t \mid \mathbf{r}_{0} ; 0\right) d \mathbf{r} .
$$

Note that $Q_{1}\left(\mathbf{r}_{0}, t ; R\right)$ depends only on the initial distance $r_{0}$ between the target and the trap because of the spherical symmetry of the former, and so we can drop the vectorial bold notation. We will also drop the subindex 0 of $r_{0}$ and use the simpler notation $Q_{1}(r, t ; R)$. Taking into account Eq. (2.8), the boundary value problem stated directly in terms of $Q_{1}(r, t ; R)$ then is [42]

$$
\begin{aligned}
\frac{\partial Q_{1}(r, t ; R)}{\partial t} & =K_{\gamma}{ }_{0} D_{t}^{1-\gamma} \nabla_{\mathbf{r}}^{2} Q_{1}(r, t ; R), \\
Q_{1}(r, 0 ; R) & =1, \\
Q_{1}(R, t ; R) & =0, \\
\lim _{r \rightarrow \infty} Q_{1}(r, t ; R) & =1 .
\end{aligned}
$$

The above problem can be solved exactly in Laplace space [42],

$$
u Q_{1}(r, u ; R)=1-\left(\frac{r}{R}\right)^{1-\frac{d}{2}} \frac{K_{d / 2-1}\left(\sqrt{r^{2} u^{\gamma} / K_{\gamma}}\right)}{K_{d / 2-1}\left(\sqrt{R^{2} u^{\gamma} / K_{\gamma}}\right)}
$$

where the $K$ 's stand for modified spherical Bessel functions of the second kind [3]. In $d=1$ and $d=3$ dimensions the Laplace transform of $Q_{1}$ can be expressed exactly in the closed form [42]

$$
\widetilde{Q}_{1}(r, u ; R)=\frac{1}{u}-\left(\frac{R}{r}\right)^{\alpha} \frac{e^{(R-r) \sqrt{u^{\gamma} / K_{\gamma}}}}{u},
$$

where $\alpha=0$ for $d=1$ and $\alpha=1$ for $d=3$. The inverse Laplace transform of $\widetilde{Q}_{1}(r, u ; R)$ can be expressed as $[24,26]$

$$
Q_{1}(r, t ; R)=Q_{1, T}(r, t ; R)=1-\left(\frac{R}{r}\right)^{\alpha} H_{11}^{10}\left[\frac{r-R}{\sqrt{K_{\gamma} t^{\gamma}}} \mid \begin{array}{l}
(1, \gamma / 2) \\
(0,1)
\end{array}\right],
$$

where

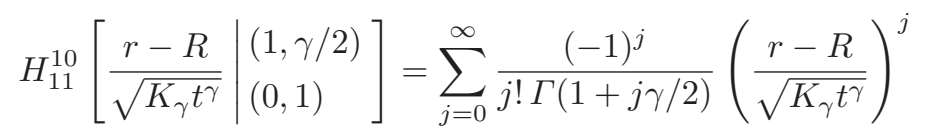

stands for a Fox $H$-function [24]. The Fox $H$-function reduces to a complementary error function when $\gamma=1$, and in this limit we recover the correct result for ordinary diffusion (see, for example, Refs. [4] and $[6])$,

$$
Q_{1, T}(r, t ; R)=1-\left(\frac{R}{r}\right)^{\alpha} \operatorname{erfc}\left(\frac{r-R}{2 \sqrt{D t}}\right),
$$

where we have adopted the usual notation for the normal diffusion coefficient $D \equiv K_{1}$. In $d=2$, there is no exact expression for the inverse Laplace transform of $\widetilde{Q}_{1}$. For long times, the use of the appropriate Tauberian theorem yields

$$
Q_{1}(r, t ; R)=Q_{1, T}(r, t ; R) \sim \frac{2}{\gamma} \frac{\ln (r / R)}{\ln \left(\alpha_{\gamma} t\right)},
$$


where $\alpha_{\gamma}=\left(4 K_{\gamma} / R^{2}\right)^{1 / \gamma}$.

The asymptotic long-time behavior of the survival probability depends strongly on dimension. In $d=1$ one easily checks that one has a power-law decay, $Q_{1, T}(r, t) \sim(r-R) / t^{\gamma / 2}$; in two dimensions, one has a slower inverse logarithmic decay, $Q_{1, T}(r, t) \propto \ln ^{-1}\left(\alpha_{\gamma} t\right)$. Finally, for $d=3$ the target has a finite survival probability, $Q_{1, T}(r, t \rightarrow \infty) \sim 1-R / r$. Asymptotically, the subdiffusive character of the particle is thus relevant only in the one-dimensional case and has only a marginal effect in $d=2$.

\subsection{The many-trap problem}

Let us now consider the problem where the target centered at $r=0$ is surrounded by $N_{0}$ randomly distributed independent point traps in a hyperspherical volume $V$. At time $t=0$ the traps begin to move according to Eq. (2.1). If any of them hits the surface of the target, both the target and the trap are instantaneously annihilated (fully absorbing target). We aim to compute the survival probability of the target $Q_{T}$ at time $t$, i.e., the probability that no traps have collided with the target up to this time. The solution of this problem can be obtained from the single-trap problem discussed in 2.1.

We take the thermodynamic limit $N_{0} \rightarrow \infty, V \rightarrow \infty$ while keeping a fixed global initial trap density $\rho_{0}=\lim _{N_{0}, V \rightarrow \infty} N_{0} / V$. Integrating over the volume exterior to the target, one then has

$$
Q_{T}(t ; R)=\lim _{N_{0}, V \rightarrow \infty}\left[\frac{1}{V} \int_{r_{0}>R} Q_{1, T}\left(\mathbf{r}_{0}, t ; R\right) d \mathbf{r}_{0}\right]^{N_{0}}=\exp \left\{-\rho_{0} R^{d} \sigma(t, R)\right\} .
$$

We have introduced the auxiliary quantity

$$
\sigma(t ; R) \equiv \frac{1}{R^{d}} \int_{r_{0}>R}\left[1-Q_{1, T}\left(\mathbf{r}_{0}, t ; R\right)\right] d \mathbf{r}_{0}=-\frac{1}{\rho_{0} R^{d}} \ln Q_{T}(t ; R)
$$

Setting $Q_{1, T}\left(\mathbf{r}_{0}, t ; R\right)=Q_{1}\left(\mathbf{r}_{0}, t ; R\right)$ and using the results of 2.1 , we can use Tauberian theorems to find the long-time behavior in the many-trap problem [42]:

$$
\sigma(t ; R) \propto\left\{\begin{array}{cl}
t^{\gamma / 2}, & d=1 \\
t^{\gamma} / \ln \left(\alpha_{\gamma} t\right), & d=2, \\
t^{\gamma}, & d=3 .
\end{array}\right.
$$

The survival probability of the target thus goes to zero in one, two and three dimensions, in contrast with the single-trap problem, where the survival probability of the target goes asymptotically to zero only in one and two dimensions, while in three dimensions the target may survive forever.

\subsection{The many-trap problem with a partially reflecting target}

Let us now consider how the behavior in the many-trap problem changes when the target is no longer fully absorbing. The starting point is once again the single-trap problem. The boundary condition Eq. (2.6) must now be replaced with the more general condition

$$
w\left(\mathbf{r}, t \mid \mathbf{r}_{0} ; 0\right)=-\Lambda \frac{\partial}{\partial n} w\left(\mathbf{r}, t \mid \mathbf{r}_{0} ; 0\right), \quad r \in \partial V .
$$

Here $\partial w / \partial n$ is the component of the gradient of $w$ perpendicular to the boundary and pointing away from the hyperspherical volume $V$ of the target, and $\Lambda \geq 0$ is a reaction rate parameter (one recovers the case of a fully absorbing target when $\Lambda=0$ ). The case $\Lambda>0$ corresponds to a partially reactive target surface that permits contact with a trap without reaction. That this is in fact the correct mathematical description of a partially reactive surface is not immediately obvious, cf. [15, 23, 35]. 
In the Laplace representation the boundary value problem for a single trap now becomes

$$
\begin{aligned}
& u \widetilde{Q}_{1}(r, u ; R)-1=u^{1-\gamma} K_{\gamma} \nabla^{2} \widetilde{Q}_{1}(r, u ; R), \\
& \widetilde{Q}_{1}(r=R, u ; R)=-\left.\Lambda \frac{\partial}{\partial n} \widetilde{Q}_{1}(r, u ; R)\right|_{r=R}, \\
& \lim _{r \rightarrow \infty} \widetilde{Q}_{1}(r, u ; R)=\frac{1}{u},
\end{aligned}
$$

where we again denote the initial distance $r_{0}$ by $r$. The result is

$$
u \widetilde{Q}_{1}(r, u)=1-\left(\frac{r}{R}\right)^{1-d / 2} \frac{K_{d / 2-1}(r z)}{K_{d / 2-1}(R z)+\Lambda z K_{d / 2}(R z)},
$$

where we have set $z=\left(u^{\gamma} / K_{\gamma}\right)^{1 / 2}$. The many-trap problem can now be solved along the same lines as in the case of a fully absorbing target. The solution was first given by Grebenkov [18]. The long-time result for the survival probability of the target can be expressed as follows:

$$
Q_{T}(t) \sim \begin{cases}\exp \left[-\frac{2 \rho_{0}}{\Gamma(1+\gamma / 2)}\left(K_{\gamma} t^{\gamma}\right)^{1 / 2}\right], & d=1, \\ \exp \left[-\frac{4 \pi \rho_{0}}{\Gamma(1+\gamma)} \frac{K_{\gamma} t^{\gamma}}{\ln \left(4 K_{\gamma} t^{\gamma} / R^{2}\right)+2 \Lambda / R}\right], & d=2, \\ \exp \left[-\frac{2 \rho_{0} R}{\Gamma(1+\gamma)(1+\Lambda / R)} K_{\gamma} t^{\gamma}\right], & d=3 .\end{cases}
$$

It is interesting to note that for long times $t$ the partially reflecting nature of the surface is not relevant for $d \leq 2$ as $\sigma(t ; R) \propto \alpha_{\gamma}^{1 / 2} / \Gamma(1+\gamma / 2)$ for $d=1$ and $\sigma(t ; R) \propto \pi \alpha_{\gamma} /\left[\Gamma(1+\gamma) \ln \left(\alpha_{\gamma}\right)\right]$ for $d=2$, both independent of $\Lambda$. However, for $d=3$, the value of $\Lambda$ appears explicitly in the characteristic decay function, i.e., $\sigma(t ; R) \propto \pi \alpha_{\gamma} /[\Gamma(1+\gamma)(1+\Lambda / R)]$.

\section{The target problem with evanescent traps}

The behavior of the survival probability of the target changes completely if an evanescence reaction is at play, i.e., if the trap or traps disappear in the course of their motion via a process which is independent of the reaction with the target. The decrease of the global trap density $\rho(t)$ due to the evanescence process is described by the rate equation

$$
\dot{\rho}(t) \equiv \frac{d \rho(t)}{d t}=-\lambda(t) \rho(t),
$$

where $\lambda(t)>0$ is a rate coefficient which in general depends on time. The solution $\rho_{0} \exp \left(-\int_{0}^{t} \lambda\left(t^{\prime}\right) d t^{\prime}\right)$ describes the decay of the trap density with time in the absence of the fully absorbing target. When $\lambda=$ constant this decay is purely exponential.

In what follows the addition of a star will distinguish these survival probabilities and associated quantities from their counterparts in the absence of trap evanescence.

\subsection{The single-trap problem}

We proceed as in the previous section and consider the situation where there is only a single trap. In order to study the survival probability of this trap, we need to combine the effects of Eqs. (3.1) and (2.1) in a reaction-subdiffusion equation $[1,25,39,40]$. A rigorous derivation starting at the level of the CTRW leads to $[1,2]$

$$
\frac{\partial w\left(\mathbf{r}, t \mid \mathbf{r}_{0}, 0\right)}{\partial t}=\frac{\rho(t)}{\rho_{0}} K_{\gamma}{ }_{0} D_{t}^{1-\gamma} \frac{\rho_{0}}{\rho(t)} \nabla_{\mathbf{r}}^{2} w\left(\mathbf{r}, t \mid \mathbf{r}_{0}, 0\right)+\frac{\dot{\rho}(t)}{\rho(t)} w\left(\mathbf{r}, t \mid \mathbf{r}_{0}, 0\right) .
$$


It is straightforward to show that the survival probability $Q_{1}^{*}\left(r_{0}, t ; R\right)=\int w\left(\mathbf{r}, t \mid \mathbf{r}_{0} ; 0\right) d \mathbf{r}$ of the trap then obeys the equation

$$
\frac{\partial Q_{1}^{*}(r, t ; R)}{\partial t}=\frac{\rho(t)}{\rho_{0}} K_{\gamma}{ }_{0} D_{t}^{1-\gamma}\left(\frac{\rho_{0}}{\rho(t)} \nabla_{\mathbf{r}}^{2}\right) Q_{1}^{*}(r, t ; R)+\frac{\dot{\rho}(t)}{\rho(t)} Q_{1}^{*}(r, t ; R),
$$

where we have again dropped the subindex from $r_{0}$ for notational convenience. The above equation must now be supplemented with the conditions

$$
\begin{aligned}
Q_{1}^{*}(r, 0 ; R) & =1, \\
Q_{1}^{*}(R, t ; R) & =0, \\
\lim _{r \rightarrow \infty} Q_{1}^{*}(r, t ; R) & =\frac{\rho(t)}{\rho_{0}} .
\end{aligned}
$$

Note that the last equation is substantially different from the corresponding equation in the absence of evanescence.

At this stage, one can easily check that the transformation $Q_{1}^{*}(r, t ; R)=\left[\rho(t) / \rho_{0}\right] \mathcal{Q}(r, t ; R)$ in Eqs. (3.3) leads to equations for the transformed function $\mathcal{Q}$ identical to the boundary value problem (2.9) for $Q_{1}$. Because of the uniqueness of the solution, we thus conclude that $\mathcal{Q} \equiv Q_{1}$, and further, to the intuitively clear relation

$$
Q_{1}^{*}(r, t ; R)=\frac{\rho(t)}{\rho_{0}} Q_{1}(r, t ; R) .
$$

Because of the evanescence of the trap, the survival probability of the trap and that of the target are no longer the same. The target has a greater survival probability than the trap, which may decay not only by interaction with the target, but also via the evanescence process. However, there is a relation between both probabilities. One can show that [2]

$$
Q_{1, T}^{*}(r, t ; R)=Q_{1}^{*}(r, t ; R)-\int_{0}^{t} Q_{1}^{*}\left(r, t^{\prime} ; R\right) \frac{\dot{\rho}\left(t^{\prime}\right)}{\rho\left(t^{\prime}\right)} d t^{\prime} .
$$

Recall that $\dot{\rho}(t) / \rho(t)<0$, and hence $Q_{1, T}^{*}(r, t ; R)>Q_{1}^{*}(r, t ; R)$, as it should be. The above equation can be used to quantify the difference between the survival probabilities.

Let us consider the particular case of an exponentially decaying trap density, $\rho(t)=\rho_{0} \exp (-\lambda t)$, where the characteristic time scale of the decay is given by $\tau \equiv \lambda^{-1}$. This could represent a unimolecular decay if this were the only decay channel, which it is not in the presence of the target. Equation (3.5) for exponential decay becomes

$$
Q_{1, T}^{*}(r, t ; R)=e^{-\lambda t} Q_{1}(r, t ; R)+\lambda \int_{0}^{t} e^{-\lambda t^{\prime}} Q_{1}\left(r, t^{\prime} ; R\right) d t^{\prime} .
$$

Taking the Laplace transform of Eq. (3.6) and using Eq. (2.10), we get

$$
u Q_{1, T}^{*}(r, u ; R)=1-\left(\frac{r}{R}\right)^{1-(d / 2)} \frac{K_{d / 2-1}\left(\sqrt{r^{2}(u+\lambda)^{\gamma} / K_{\gamma}}\right)}{K_{d / 2-1}\left(\sqrt{R^{2}(u+\lambda)^{\gamma} / K_{\gamma}}\right)} .
$$

Equation (3.7) can be used to obtain the long-time behavior of $Q_{1, T}^{*}(r, t ; R)$ in arbitrary Euclidean dimension via the final value theorem for the Laplace transform. One has

$$
Q_{1, T}^{*}(r, \infty ; R)=\lim _{u \rightarrow 0} u \tilde{Q}_{1, T}^{*}(r, u ; R)=1-\left(\frac{r}{R}\right)^{1-(d / 2)} \frac{K_{d / 2-1}\left(\sqrt{r^{2} \lambda^{\gamma} / K_{\gamma}}\right)}{K_{d / 2-1}\left(\sqrt{R^{2} \lambda^{\gamma} / K_{\gamma}}\right)} .
$$

In one and three dimensions, Eq. (3.7) becomes

$$
\tilde{Q}_{1, T}^{*}(r, u ; R)=\frac{1}{u}-\left(\frac{R}{r}\right)^{\alpha} \frac{e^{-(r-R) \sqrt{(u+\lambda)^{\gamma} / K_{\gamma}}}}{u}
$$


with $\alpha=0$ for $d=1$ and $\alpha=1$ for $d=3$. Multiplying this equation by the Laplace variable $u$, taking the limit $u \rightarrow 0$ and applying the final value theorem we get the asymptotic survival probability

$$
Q_{1, T}^{*}(r, \infty ; R)=1-\left(\frac{R}{r}\right)^{\alpha} e^{-2(r-R) / \ell_{\gamma}}
$$

where

$$
\ell_{\gamma}=\left(4 K_{\gamma} \tau^{\gamma}\right)^{1 / 2}
$$

is directly related to the typical distance travelled by the trap during its mean survival time $\tau$ in the absence of the target. Thus, for any non-zero value of $\lambda=\tau^{-1}$ the survival probability of the target is finite. However, for $d=1$ this survival probability vanishes as $\lambda \rightarrow 0$ because of the recurrence properties of one-dimensional random walks [21]. The probability $1-Q_{1, T}^{*}(r, \infty ; R)$ that the target is eventually annihilated is seen to decrease exponentially with the separation distance $r-R$ between the trap and the surface of the target.

In the normal diffusive case $\gamma=1$, one can find the inverse Laplace transform of the right hand side in Eq. (3.9). The result can be expressed as follows:

$$
\begin{aligned}
Q_{1, T}^{*}(r, t ; R)= & -\frac{1}{2}\left(\frac{R}{r}\right)^{\alpha} e^{\sqrt{\lambda(r-R)^{2} / D}} \operatorname{erfc}\left(\sqrt{\frac{(r-R)^{2}}{4 D t}}+\sqrt{\lambda t}\right) \\
& -\frac{1}{2}\left(\frac{R}{r}\right)^{\alpha} e^{-\sqrt{\lambda(r-R)^{2} / D}} \operatorname{erfc}\left(\sqrt{\frac{(r-R)^{2}}{4 D t}}-\sqrt{\lambda t}\right) .
\end{aligned}
$$

When $\gamma<1$, no exact expression for the integral in (3.6) is available. However, using the appropriate long-time expansions one finds that for large $t$

$$
Q_{1, T}^{*}(r, \infty ; R)-Q_{1, T}^{*}(r, t ; R) \sim-\left(\frac{R}{r}\right)^{\alpha} \frac{1}{\Gamma\left(1-\frac{\gamma}{2}\right)} \frac{r-R}{\lambda^{1+\frac{\gamma}{2}} \ell_{\gamma}} t^{-1-\frac{\gamma}{2}} e^{-\lambda t} .
$$

For $d=2$ the inverse transform of the right hand side of Eq. (3.7) can no longer be reduced to elementary functions. However, one can obtain the stationary survival probability by setting $d=2$ in Eq. (3.8),

$$
Q_{1, T}^{*}(r, t \rightarrow \infty ; R)=1-\frac{K_{0}\left(2 r / \ell_{\gamma}\right)}{K_{0}\left(2 R / \ell_{\gamma}\right)} .
$$

Taking into account the fact that

$$
Q_{1, T}^{*}(r, t ; R)=Q_{1, T}^{*}(r, \infty ; R)+e^{-\lambda t} Q_{1}(r, t ; R)-\lambda \int_{t}^{\infty} e^{-\lambda t^{\prime}} Q_{1}\left(r, t^{\prime} ; R\right) d t^{\prime}
$$

and using the long time expansion given by Eq. (2.15) one gets

$$
\begin{aligned}
Q_{1, T}^{*}(r, t ; R) & \approx Q_{1, T}^{*}(r, \infty ; R)+\frac{2}{\gamma} \ln \left(\frac{r}{R}\right) \int_{t}^{\infty} d t^{\prime} \frac{e^{-\lambda t^{\prime}}}{t^{\prime} \ln ^{2}\left(\alpha_{\gamma} t^{\prime}\right)}, \quad t \rightarrow \infty \\
& \approx Q_{1, T}^{*}(r, \infty ; R)+\frac{2}{\gamma} \ln \left(\frac{r}{R}\right) \frac{e^{-\lambda t}}{\lambda t \ln ^{2}\left(\alpha_{\gamma} t\right)}\left[1+\frac{2+\ln \left(\alpha_{\gamma} t\right)}{\lambda t \ln \left(\alpha_{\gamma} t\right)}+\cdots\right],
\end{aligned}
$$

where we have repeatedly applied partial integration to go from the first to the second line. To leading order, the approach to the steady state is dominated by an exponential multiplied by a slowly decreasing second-order logarithmic correction. 


\subsection{The many-trap problem}

In the presence of a collection of evanescent traps, the statistical independence of the traps is not affected by the evanescence reaction. The survival probability $Q_{T}^{*}(t ; R)$ of the target at time $t$ can therefore be computed by following the same strategy as in the absence of evanescence. Equations (2.16) and (2.17) are now respectively replaced with

$$
Q_{T}^{*}(t ; R)=\lim _{N_{0}, V \rightarrow \infty}\left[\frac{1}{V} \int_{r>R} Q_{1, T}^{*}(r, t ; R) d \mathbf{r}\right]^{N_{0}}=\exp \left\{-\rho_{0} R^{d} \sigma^{*}(t, R)\right\},
$$

where

$$
\sigma^{*}(t ; R)=\frac{1}{R^{d}} \int_{r>R}\left[1-Q_{1, T}^{*}(r, t ; R)\right] d \mathbf{r}=-\frac{1}{\rho_{0} R^{d}} \ln Q_{T}^{*}(t ; R) .
$$

We have now clarified the relation between $Q_{1}^{*}(r, t ; R)$ (survival probability of a single evanescent trap) and $Q_{1}(r, t ; R)$ (survival probability of a single non-evanescent trap) and between $Q_{1, T}^{*}(r, t ; R$ ) (survival probability of the target in the presence of a single evanescent trap) and $Q_{1}^{*}(r, t ; R)$ (survival probability of a single evanescent trap). We can now use these relations and proceed to the many-trap problem. In Ref. [2] we showed that starting with Eq. (3.18) and using the fractional equation Eq. (3.3a) together with Eq. (3.4) and Eq. (3.5) we arrive at

$$
\sigma^{*}(t ; R)=S_{d} \frac{K_{\gamma}}{R} \int_{0}^{t}\left[\left.{ }_{0} D_{t^{\prime}}^{1-\gamma} \frac{\partial Q_{1}\left(r, t^{\prime} ; R\right)}{\partial r}\right|_{r=R}\right] \frac{\rho\left(t^{\prime}\right)}{\rho_{0}} d t^{\prime},
$$

where $S_{d}=2 \pi^{d / 2} / \Gamma(d / 2)$ denotes the surface of a $d$-dimensional hypersphere of unit radius. The formula (3.20) relates the logarithm of the survival probability of the target, now in the presence of a collection of evanescent traps, to the survival probability of the target in the presence of a single non-evanescent trap. If the trap density $\rho(t)$ decays sufficiently rapidly, then we can conclude that $Q_{1, T}^{*}(t \rightarrow \infty, R)>0$ and $\sigma^{*}(t \rightarrow \infty ; R)<\infty$, which allows us to refer to Eq. (3.20) to arrive at the large- $t$ result

$$
\sigma^{*}(\infty ; R)-\sigma^{*}(t ; R)=S_{d} \frac{K_{\gamma}}{R} \int_{t}^{\infty}\left[\left.{ }_{0} D_{t^{\prime}}^{1-\gamma} \frac{\partial Q_{1}\left(r, t^{\prime} ; R\right)}{\partial r}\right|_{r=R}\right] \frac{\rho\left(t^{\prime}\right)}{\rho_{0}} d t^{\prime} .
$$

We next need to specify the dimensionality as well as the explicit time-dependent form of the trap density. Using the one-dimensional form of $Q_{1}$ (Eq. (2.12) with $\alpha=0$ ) in Eq. (3.20) we get after evaluation of the corresponding fractional derivative

$$
\sigma^{*}(t ; R)=\frac{1}{\rho_{0} R} \frac{2 \sqrt{K_{\gamma}}}{\Gamma(\gamma / 2)} \int_{0}^{t} \rho\left(t^{\prime}\right) t^{\prime \gamma / 2-1} d t^{\prime}
$$

Similarly, in the three-dimensional case we find from Eq. (2.12) (with $\alpha=1$ ) and Eq. (3.20) [2]

$$
\sigma^{*}(t ; R)=\frac{4 \pi \sqrt{K_{\gamma}}}{\rho_{0} R \Gamma(\gamma / 2)} \int_{0}^{t} \rho\left(t^{\prime}\right) t^{\prime \gamma / 2-1} d t^{\prime}+\frac{4 \pi K_{\gamma}}{\rho_{0} R^{2} \Gamma(\gamma)} \int_{0}^{t} \rho\left(t^{\prime}\right) t^{\prime \gamma-1} d t^{\prime} .
$$

Let us further assume that the trap density $\rho(t)$ decays sufficiently rapidly to ensure that $\sigma^{*}(\infty ; R)$ is finite. From Eq. (3.21) and Eq. (2.15) we find that for $d=2$,

$$
\sigma^{*}(\infty ; R)-\sigma^{*}(t ; R) \sim \frac{4 \pi K_{\gamma}}{\rho_{0} R^{2} \Gamma(\gamma+1)} \int_{t}^{\infty} \frac{\rho\left(t^{\prime}\right) t^{\prime \gamma-1}}{\ln \left(\alpha_{\gamma} t^{\prime}\right)} d t^{\prime}
$$




\subsubsection{Exponential evanescence}

For a trap density decay of the form $\rho(t)=\rho_{0} e^{-\lambda t}$, the computation of the final value of $\sigma^{*}(t ; R)$ in arbitrary integer dimensions is readily obtained from the Laplace transformed quantity $\sigma^{*}(u ; R)[2]$. The Laplace transform of Eq. (3.19) immediately leads to

$$
\sigma^{*}(u ; R)=\frac{S_{d} K_{\gamma}^{1 / 2}}{u R(u+\lambda)^{\gamma / 2}} \frac{K_{d / 2}\left(\sqrt{R^{2}(u+\lambda)^{\gamma} / K_{\gamma}}\right)}{K_{d / 2-1}\left(\sqrt{R^{2}(u+\lambda)^{\gamma} / K_{\gamma}}\right)} .
$$

The final value theorem then gives

$$
\sigma^{*}(\infty ; R)=\lim _{u \rightarrow 0} u \sigma^{*}(u ; R)=S_{d} \frac{\ell_{\gamma}}{2 R} \frac{K_{d / 2}\left(2 R / \ell_{\gamma}\right)}{K_{d / 2-1}\left(2 R / \ell_{\gamma}\right)} .
$$

For odd dimensions, Eq. (3.26) can be conveniently rewritten as rational functions of the argument $R / \ell_{\gamma}$. For example, $\sigma^{*}(\infty ; R)=\ell_{\gamma} / R$ for $d=1$ and $\sigma^{*}(\infty ; R)=\left(2 \pi \ell_{\gamma} / R\right)\left(1+\ell_{\gamma} / 2 R\right)$ for $d=3$.

Obtaining exact expressions for the survival probability at finite times is more difficult. For $d=1$ one can get an exact expression due to the simplification of the Bessel functions appearing in the Laplace transform,

$$
\sigma^{*}(t ; R)=\frac{\ell_{\gamma}}{R}\left(1-\frac{\Gamma(\gamma / 2, \lambda t)}{\Gamma(\gamma / 2)}\right) .
$$

Eq. (3.27) is equivalent to Eq. (15) in Ref. [44]. For $d=3$ we find

$$
\sigma^{*}(t ; R)=2 \pi \frac{\ell_{\gamma}}{R}\left(1-\frac{\Gamma(\gamma / 2, \lambda t)}{\Gamma(\gamma / 2)}\right)+\pi \frac{\ell_{\gamma}^{2}}{R^{2}}\left(1-\frac{\Gamma(\gamma, \lambda t)}{\Gamma(\gamma)}\right),
$$

resulting in a smaller survival probability than in $d=1$. As noted already, there are no simple, closedform solutions valid for arbitrary times for $d=2$, although we are able to extract some limiting behaviors for this case as well (see below).

The explicit asymptotic results for the approach to the final values for $d=1$ and $d=3$ respectively follow from Eqs. (3.27) and (3.28), and for $d=2$ the result can be inferred from Eq. (3.24). The results hold for $\gamma \leq 1$, that is, also for normal diffusion:

$$
\sigma^{*}(\infty ; R)-\sigma^{*}(t ; R) \propto\left\{\begin{array}{cc}
t^{\gamma / 2-1} e^{-\lambda t}, & d=1, \\
\ln ^{-1}\left(\alpha_{\gamma} t\right) t^{\gamma-1} e^{-\lambda t}, & d=2, \\
t^{\gamma-1} e^{-\lambda t}, & d=3 .
\end{array}\right.
$$

The decay of the survival probability to the final state shown in Eq. (3.29) is seen to be faster as the dimensionality increases. It is also straightforward to show that the long time behavior prescribed by Eq. (3.29) for the $d=3$ case remains valid for $d>3$ with a $d$-dependent prefactor.

Interestingly, the limit $\lambda \rightarrow 0$ (no evanescence) turns out to be singular. Indeed, taking this limit in Eq. (3.29) does not yield the correct result displayed in Eq. (2.18). We conclude that the evanescence reaction completely changes the physics of the problem, affecting both the steady state and the decay form of the survival probability.

\subsubsection{Power-law evanescence}

We next focus on a power-law decaying density,

$$
\rho(t)=\frac{\rho_{0}}{(1+t / \tau)^{\beta}}, \quad \beta>0 .
$$

One might think of this time dependence as arising from some higher-order kinetics associated with the linear differential equation (3.1) together with a proper choice of the exponent $\beta$. The survival probability 
of the target in this case depends not only on dimensionality but on the relative values of the power-law decay exponent $\beta$ and the subdiffusion exponent $\gamma$.

In one dimension, $d=1$, the outcome follows directly from Eq. (3.22), and can be summarized as follows [44]:

$$
\sigma^{*}(t ; R) \sim \begin{cases}\ell_{\gamma} \frac{\Gamma(\beta-\gamma / 2)}{R \Gamma(\beta)}, & \beta>\gamma / 2, \\ \frac{\ell_{\gamma}}{R \Gamma(\gamma / 2)} \ln (t / \tau), & \beta=\gamma / 2, \\ \frac{\ell_{\gamma}}{R(\gamma / 2-\beta) \Gamma(\gamma / 2)}(t / \tau)^{\gamma / 2-\beta}, & \beta<\gamma / 2,\end{cases}
$$

where $\ell_{\gamma}$ is defined as in the exponential case, i.e., $\ell_{\gamma}=\left(4 K_{\gamma} \tau^{\gamma}\right)^{1 / 2}$.

Next we consider the two-dimensional system. We shall distinguish three different cases. Additional details of the calculations can be found in [2].

Case $1(\beta>\gamma)$. In this case the target has a finite probability of eternal survival. Using the explicit form of $\rho\left(t^{\prime}\right)$ in Eq. (3.24) we find

$$
\sigma^{*}(\infty ; R)-\sigma^{*}(t ; R) \sim \frac{\pi \ell_{\gamma}^{2}}{(\beta-\gamma) \Gamma(\gamma+1) R^{2}} \frac{(t / \tau)^{\gamma-\beta}}{\ln \left(\alpha_{\gamma} t\right)} .
$$

Unfortunately, it does not seem possible to find an explicit exact expression for $\sigma^{*}(\infty ; R)$ due to the lack of an exact expression for $Q_{1}\left(r, t^{\prime} ; R\right)$ valid for the whole time domain.

Case $2(\beta=\gamma)$. In this marginal case the target also eventually disappears, but with a different analytic approach to the empty state:

$$
\sigma^{*}(t ; R) \sim \frac{\pi \ell_{\gamma}^{2}}{\Gamma(\gamma+1) R^{2}} \ln \left(\ln \left(\alpha_{\gamma} t\right)\right)
$$

Thus, the target is eventually killed with certainty, in agreement with the result given in Ref. [10] for the special case $\beta=\gamma=1$ (normal diffusive traps).

Case $3(\beta<\gamma)$. In this case the long time behavior is given by

$$
\sigma^{*}(t ; R) \sim \frac{\pi \ell_{\gamma}^{2}}{(\gamma-\beta) \Gamma(\gamma+1) R^{2}} \frac{(t / \tau)^{\gamma-\beta}}{\ln \left(\alpha_{\gamma} t\right)} .
$$

Hence when $\beta<\gamma$ the target eventually disappears with certainty.

We next turn to the three dimensional case. There are again three relevant subcases [2].

Case $1(\beta>\gamma)$.

$$
\sigma^{*}(t ; R) \sim \sigma^{*}(\infty ; R)-\frac{\pi \ell_{\gamma}^{2}}{R^{2}} \frac{(t / \tau)^{\gamma-\beta}}{(\beta-\gamma) \Gamma(\gamma)}
$$

with

$$
\sigma^{*}(\infty ; R)=2 \pi \frac{\ell_{\gamma}}{R} \frac{\Gamma(\beta-\gamma / 2)}{\Gamma(\beta)}+\frac{\pi \ell_{\gamma}^{2}}{R^{2}} \frac{\Gamma(\beta-\gamma)}{\Gamma(\beta)},
$$

leading to a non-zero survival probability $Q_{T}^{*}(\infty ; R)=\exp \left[-\rho_{0} R^{2} \sigma^{*}(\infty ; R)\right]$.

Case $2(\beta=\gamma)$. In this case, the survival probability $Q_{T}^{*}(t ; R)$ goes to zero as $(t / \tau)^{-\pi \rho_{0} R \ell_{\gamma}^{2} / \Gamma(\gamma)}$, that is, $\sigma^{*}(t ; R) \propto \ln (t / \tau)$.

Case $3(\beta<\gamma)$. In this case one has $Q_{T}^{*}(t ; R) \propto \exp \left(-C t^{\gamma-\beta}\right)$ with $C>0$, that is, $\sigma^{*}(t ; R) \propto t^{\gamma-\beta}$. Thus, in two and three dimensions the target has a finite probability of eternal survival only for $\beta>\gamma$. For comparison, in the one-dimensional case it was found that the target has a chance of eternal survival only when $\beta>\gamma / 2$ [cf. Eq. (3.31)]. We thus see that the interplay between subdiffusive transport and the evanescence reaction in higher dimensions also determines whether the target can ultimately survive. 


\section{Summary and Outlook}

We have presented some recent results on the survival probability of an immobile target in the presence of a single diffusive or subdiffusive trap and also in the presence of noninteracting diffusive or subdiffusive point traps. We have considered both the case where the traps are non-evanescent and also when they are subject to the evanescence reaction.

Our conclusions can be summarized as follows. In the single-trap problem without evanescence, the subdiffusive character of the trap is of relevance for the long-time survival probability only in the onedimensional case; its influence in the two-dimensional case is marginal, whereas in the three-dimensional case it does not affect the asymptotic value of $Q_{1, T}$.

In the many-trap problem without evanescence, the decay of the survival probability of the target at long times is slowed down with respect to the case of normal diffusion in one, two and three dimensions. In all three cases, one has a stretched exponential form which depends on the anomalous diffusion exponent $\gamma$ (the usual logarithmic correction appears in $d=2$ ). In the many-trap problem with a finite reaction rate, the reflecting nature of the target manifests itself mainly in three dimensions. In all cases, the behavior of the rate constant $k(t)$ describing the velocity of the reaction between the target and the traps can be easily obtained by comparing Eq. (1.2) with Eq. (2.16). This comparison leads to the relation $k(t)=\rho_{0} R^{d} d \sigma(t, R) / d t$.

The evanescence reaction was found to completely modify the behavior of the single-trap and the many-trap problems, both at the level of the steady state and of the decay of the survival probability to a finite steady state or to zero. In the single-trap problem with exponentially decaying trap density $\rho(t)=\rho_{0} e^{-\lambda t}$ the target has a finite survival probability in all dimensions. The general expression of this probability involves a ratio of modified Bessel functions, which reduces to exponential functions in one and three dimensions. In these cases, the approach to the steady state is given by an exponential times an inverse power of $t$ with a $\gamma$-dependent exponent. In two dimensions the decay is even more complex, as it also involves the square of an inverse logarithm. In particular, our results hold also for the normal diffusion case $\gamma=1$.

In the many-trap problem with evanescence, general expressions for the characteristic decay function $\sigma^{*}(t ; R)$ as a function of the time dependent trap density $\rho(t)$ can be obtained. In the special case of exponential evanescence, we find like in the single-trap problem that there is a finite survival probability of the target in all dimensions because the traps die sufficiently quickly in their search of the target. By way of contrast, the target has a zero survival probability in all dimensions when the traps do not evanesce. The long-time approach to the final value of the survival probability turns out to be more complex than in the case of non-evanescent traps, and in the subdiffusive case $\gamma<1$ it involves powers of $t$ as well as exponential factors $e^{-\lambda t}$ (with a logarithmic correction in $d=2$ ). On the other hand, when the density decays as a power law, $\rho(t) \propto t^{-\beta}$ with $\beta>0$, the behavior depends on the relative values of $\beta$ and the anomalous diffusion exponent $\gamma$ of the traps. In one dimension, the target has a finite asymptotic survival probability if $\beta>\gamma / 2$, whereas in two and three dimensions the target only has a finite chance of eternal survival when $\beta>\gamma$.

As already mentioned in the Introduction, the general case where not only the traps but also the target moves (either diffusively or subdiffusively) is difficult to deal with, even in the absence of the evanescence reaction (the case of a normal diffusive target and normal diffusive evanescent traps has been considered in Ref. [20]). The main difficulty is that in such a case the respective distances between the target and each of the traps no longer evolve as independent variables. In some cases, the most useful approximations are based on the fact that at long times the dominant contribution to the survival probability comes from the subset of trajectories where the target remains immobile $[7,9,28,41,43]$. Ultimately, this behavior finds its roots in what has been labeled the "Pascal principle", apparently first formulated for the process of migration-accelerated quenching of incoherent excitations in Ref. [11] and widely used in the literature on diffusion-controlled reactions ever since (see e.g. $[12,27,41]$ ). The Pascal principle states that a target 
placed in a symmetric initial distribution of traps survives longer on average if it remains immobile. ${ }^{1}$ It is reasonable to assume that approximations based on this fact might also work in the presence of the evanescence reaction.

Finally, it should be clear from the discussion in the Introduction that any process disabling the interaction between the target and the traps can be regarded as an evanescence reaction in the framework of the target and the trapping problems. However, in real systems such processes are often reversible, thus making extensions of the theory to account for reversible evanescence processes interesting both from a theoretical and a practical point of view. In particular, Refs. [5] and [6] consider models where either the target or the traps can switch between an active state, which enables the interaction between the two particle species, and an inactive state where such interaction is precluded. Many of the arguments used should remain applicable to the case of subdiffusive motion, thereby making the extended problem with subdiffusive species amenable to analytical solution.

Acknowledgements. This work was partially funded by the Ministerio de Ciencia y Tecnología (Spain) through Grant No. FIS2010-16587 (partially financed by FEDER funds), by the Junta de Extremadura through Grant. No. GRU10158, and by the US National Science Foundation Grant No. PHY-0855471.

\section{References}

[1] E. Abad, S. B. Yuste, K. Lindenberg. Reaction-subdiffusion and reaction-superdiffusion equations for evanescent particles performing continuous-time random walks. Phys. Rev. E 81 (2010), 031115.

[2] E. Abad, S.B. Yuste, K. Lindenberg. Survival probability of an immobile target in a sea of evanescent diffusive or subdiffusive traps: A fractional equation approach.. Phys. Rev. E 86 (2012), 061120.

[3] M. Abramowitz, I. A. Stegun. Handbook of Mathematical Functions. Dover, New York, 1965.

[4] A. V. Barzykin, M. Tachiya. Diffusion-influenced reaction kinetics on fractal structures. J. Chem. Phys. 99 (1993), 9591-9597.

[5] O. Bénichou, M. Moreau, G. Oshanin. Kinetics of stochastically gated diffusion-limited reactions and geometry of random walk trajectories. Phys. Rev. E 61 (2000), 3388-3406.

[6] A. M. Berezhkovskii, D.-Y. Yang, S. H. Lin, Yu. A. Makhnovskii, S.-Y. Sheu. Smoluchowski-type theory of stochastically gated diffusion-influenced reactions. J. Chem. Phys. 106 (1997), 6985-6998.

[7] R. Borrego, E. Abad, S.B. Yuste. Survival probability of a subdiffusive particle in a d-dimensional sea of mobile traps. Phys. Rev. E 80 (2009), 061121.

[8] M. Bramson, J. L. Lebowitz. Asymptotic Behavior of Densities in Diffusion-Dominated Annihilation Reactions. Phys. Rev. Lett. 61 (1988), 2397-2400. M. Bramson, J. L. Lebowitz. Asymptotic behavior of densities for two-particle annihilating random walks. J. Stat. Phys. 62 (1991), 297-372.

[9] A. J. Bray, R. A. Blythe. Exact Asymptotics for One-Dimensional Diffusion with Mobile Traps. Phys. Rev. Lett. 89 (2002), 150601.

[10] A.J. Bray, S. N. Majumdar, R. A. Blythe. Formal solution of a class of reaction-diffusion models: Reduction to a single-particle problem. Phys. Rev. E 67 (2003), 060102(R).

[11] S. F. Burlatsky, G. Oshanin, A. A. Ovchinnikov. Fluctuation induced kinetics of incoherent excitations quenching. Phys. Lett. A 139 (1989), 245-248.

[12] L-C. Chen, R. Sun, A Monotonicity Result for the Range of a Perturbed Random Walk. arXiv:1203.1389v2 [math.PR]

[13] F. C. Collins, G. E. Kimball. Diffusion-controlled reaction rates. J. Colloid. Sci. 4 (1949), 425-437.

[14] D. V. Donsker, S. R. S. Varadhan. Asymptotics for the Wiener sausage. Commun. Pure Appl. Math. 32 (1975), 525565 ; D. V. Donsker and S. R. S. Varadhan. On the number of distinct sites visited by a random walk. Commun. Pure Appl. Math. 32 (1979), 721-747.

[15] J. D. Eaves, D. R. Reichman. The subdiffusive targeting problem. J. Phys. Chem. B 112 (2008), 4283-4289.

[16] J. Franke, S. Majumdar. Survival probability of an immobile target surrounded by mobile traps. J. Stat. Mech. (2012) P05024.

[17] S. H. Glarum. Dielectric Relaxation of Isoamyl Bromide. J. Chem. Phys. 33 (1960), 639-643.

[18] D. S. Grebenkov. Searching for partially reactive sites: Analytical results for spherical targets. J. Chem. Phys. 132 (2010), 034104.

[19] B. I. Henry, T. A. M. Langlands, S. L. Wearne. Anomalous diffusion with linear reaction dynamics: From continuous time random walks to fractional reaction-diffusion equations. Phys. Rev. E 74 (2006), 031116.

[20] F. den Hollander, K.E. Shuler. Random walks in a random field of decaying traps. J. Stat. Phys. 67 (1992), $13-31$.

[21] B. H. Hughes. Random Walks and Random Environments. Volume 1: Random Walks. Clarendon Press, Oxford, 1995.

\footnotetext{
${ }^{1}$ This statement is of course true only when the motions of the target and the traps are uncorrelated. However, the case with non-trivial correlations between the target and the traps can also be dealt with analytically, see Ref. [29].
} 
[22] J. Kim, Y. Jung, J. Jeon, S. Lee. Diffusion-influenced radical recombination in the presence of a scavenger. J. Chem. Phys. 104 (1996), 5784-5797.

[23] M. A. Lomholt, I. M. Zaid, R. Metzler. Subdiffusion and Weak Ergodicity Breaking in the Presence of a Reactive Boundary. Phys. Rev. Lett. 98 (2007), 200603.

[24] A. M. Mathai, R. K. Saxena. The H-function with Applications in Statistics and Other Disciplines. Wiley, New York, 1978.

[25] See ch. 3 in V. Méndez, S. Fedotov, W. Horsthemke. Reaction-Transport Systems: Mesoscopic Foundations, Fronts, and Spatial Instabilities. Springer, Berlin, 2010.

[26] R. Metzler, J. Klafter. The random walk's guide to anomalous diffusion: a fractional dynamics approach. Phys. Rep. 339 (2000), 1-77.

[27] M. Moreau, G. Oshanin, O. Bénichou, M. Coppey. Pascal principle for diffusion-controlled trapping reactions. Phys. Rev. E 67 (2003), 045104(R).

[28] G. Oshanin, O. Bénichou, M. Coppey, M. Moreau. Trapping reactions with randomly moving traps: Exact asymptotic results for compact exploration. Phys. Rev. E 66 (2002), 060101(R).

[29] G. Oshanin, O. Vasilyev, P. L. Krapivsky, J. Klafter. Survival of an evasive prey. PNAS 106 (2009), 13696-13701.

[30] I. Podlubny. Fractional Differential Equations. Academic Press, San Diego, 1999.

[31] S. A. Rice. Diffusion-limited Reactions. Elsevier. Amsterdam, 1985.

[32] J. J. Ruiz-Lorenzo, S. B. Yuste, K. Lindenberg. Simulations for trapping reactions with subdiffusive traps and subdiffusive particles. J. Phys.: Condens. Matter. 19 (2007), 065120.

[33] H. Sano, M. Tachiya. Partially diffusion controlled recombination. J. Chem. Phys. 71 (1979), 1276-1282.

[34] K. Seki, A. I. Shushin, M. Wojcik, M. Tachiya. Specific features of the kinetics of fractional-diffusion assisted geminate reactions. J. Phys.: Condens. Matter 19 (2007), 065117.

[35] K. Seki, M. Wojcik, M. Tachiya. Fractional reaction-diffusion equation. J. Chem. Phys. 119 (2003), $2165-2170$.

[36] V. P. Shkilev. Subdiffusion with the Disappearance of Particles at the Time of a Jump. J. Exp. Theor. Phys. (Springer) 112 (2011), 1071-1076.

[37] M. F. Shlesinger, E. W. Montroll. On the Williams-Watts function of dielectric relaxation. PNAS 81 (1984), 1280-1283.

[38] M. Smoluchowski. Versuch einer mathematischen Theorie der Koagulationskinetik kolloider Lösungen. Z. Phys. Chem. 92 (1917), 129-168.

[39] I. M. Sokolov, M. G. W. Schmidt, F. Sagués. Reaction-subdiffusion equations. Phys. Rev. E 73 (2006), 031102; B. I. Henry, T. A. M. Langlands, S. L. Wearne. Anomalous diffusion with linear reaction dynamics: From continuous time random walks to fractional reaction-diffusion equations. Phys. Rev. E 74 (2006), 031116; A. Yadav, W. Horsthemke. Kinetic equations for reaction-subdiffusion systems: Derivation and stability analysis. Phys. Rev. E 74 (2006), 066118; M. G. W. Schmidt, F. Sagués, I. M. Sokolov. Mesoscopic description of reactions for anomalous diffusion: a case study. J. Phys.: Condens. Matter 19 (2007) 065118; D. Froemberg, I. M. Sokolov. Stationary Fronts in an A+B $\rightarrow 0$ Reaction under Subdiffusion. Phys. Rev. Lett. 100 (2008), 108304; A. Yadav, S. M. Milu, W. Horsthemke. Turing instability in reaction-subdiffusion systems. Phys. Rev. E 78 (2008), 026116.

[40] S. B. Yuste, E. Abad, K. Lindenberg. Reactions in Subdiffusive Media and Associated Fractional Equations in Fractional Dynamics. Recent Advances. J. Klafter, S. C. Lim, and R. Metzler (Eds.). World Scientific, Singapore, 2011.

[41] S. B. Yuste, K. Lindenberg. Trapping reactions with subdiffusive traps and particles characterized by different anomalous diffusion exponents. Phys. Rev. E 72 (2005), 061103.

[42] S. B. Yuste, K. Lindenberg. Subdiffusive target problem: Survival probability. Phys. Rev. E 76 (2007), 051114.

[43] S. B. Yuste, G. Oshanin, K. Lindenberg, O. Bénichou, J. Klafter. Survival probability of a particle in a sea of mobile traps: A tale of tails. Phys. Rev. E 78 (2008), 021105.

[44] S. B. Yuste, J. J. Ruiz-Lorenzo, K. Lindenberg. Target problem with evanescent subdiffusive traps. Phys. Rev. E 74 (2006), 046119. 